\title{
Los modelos logísticos como herramientas para la construcción de la eficiencia empresarial
}

\section{Logistics models as tools for the construction of business efficiency}

Recibido: 2 de febrero de 2017

Evaluado: 22 de marzo de 2017

Aceptado: 7 de mayo de 2017

\section{Eduardo Norman Acevedo (Colombia)}

Magister en Gerencia Estratégica de Mercadeo. Institución Universitaria Politécnico Grancolombiano

ednorman@poligran.edu.co

\section{Ángela Julieta Mora Ramírez (Colombia)}

Magister en Educación. Docente de la Institución Universitaria Politécnico Grancolombiano amoraram@poligran.edu.co

\section{Resumen:}

Mediante una vigilancia de literatura, buscamos definir la aparición de la globalización tecnológica, lo cual hace necesario adoptar buenas prácticas empresariales que permitan a las empresas alcanzar mejores resultados y mayores índices de eficiencia para los diferentes grupos de interés. En este artículo, se propone metodológicamente un análisis de literatura, con el fin de revisar las tendencias en la cadena de valor y la logística administrativa partiendo de propuestas de logística como justo a tiempo, la cual requiere una actualización al contexto global. Los hallazgos de este artículo podrían dar luces a factores clave para el éxito empresarial. La mayor parte de los artículos consultados coinciden en que, al enfrentar los retos impuestos por la globalización, el posicionamiento de las empresas puede ser aprovechado para lograr una ventaja competitiva.

Palabras Clave: logística empresarial; cadena de valor; globalización; cambio tecnológico; operaciones; producción; gestión

\section{Summary:}

Through a literature monitoring process we seek to define the emergence of technological globalization, which makes it necessary to adopt good business practices that allow companies to achieve better results and higher efficiency rates for the related interest groups. In this article, a literature analysis is proposed as a methodology in order to review trends in the value chain and administrative logistics based on logistics proposals such as the "Just in Time" model, which requires updating towards the global context. The findings of this article could shed light on key factors for business success. Most of the articles consulted agree that by facing the challenges imposed with globalization, positioning of companies can be exploited to achieve a competitive advantage.

Keywords: Logistic, value chain, globalization, technologic change, operations, production, management. 


\section{Introducción}

La industrialización, cerca de la década de 1970 , permite a los japoneses analizar su desarrollo económico fruto de la industria creciente, cuestionando sus procesos de producción para volverlos más eficientes. Surgen, entonces, procesos que plantean el reto de maximizar los elementos restringidos de su producción e insumos. Así es como una técnica innovadora propuesta por Ohno (1988), conocida como justo a tiempo y desarrollada para Toyota, identifica la flexibilidad de los procesos y concibe la producción como un todo y las posibilidades en producción orientadas al objetivo.

La propuesta de este modelo por parte de Hay (2002) se concibe como la adaptación de estos postulados a los procesos productivos actuales. En este artículo, analizaremos a la luz de la literatura las variantes propuestas a este modelo y las adaptaciones y los aportes que este hizo a la logística durante la etapa de producción.

Durante la revisión de literatura, se encuentra que los conceptos del modelo justo a tiempo son adaptables también al proceso de distribución física. Así es como se plantea el gran parecido con el lead time propuesto por (Ballou, 2004), donde se analizan las unidades de tiempo entre la orden de compra y cuando material ingresa a bodega para iniciar la producción. Esta pequeña diferencia se considera una ventaja competitiva, es decir, el uso de la información para la reducción de la incertidumbre y un manejo eficiente, particularmente en los inventarios, y la apropiación de tecnología a fin de optimizar la producción y, por consiguiente, la atención de clientes.

El concepto de lead time también es adaptable por medio de información electrónica para reducir el tiempo del ciclo del pedido. Combinar tecnología para optimizar el proceso de producción, así como el servicio relacional con el cliente, son cruciales a fin de lograr una reducción de costos.
Los postulados objetivos del modelo le transmiten los resultados al usuario final, teniendo en cuenta que busca la satisfacción total del cliente. Pero siempre manteniendo los mejores estándares de producción, donde la empresa no pierde, en un constante equilibrio, por medio de un proceso de aprendizaje tanto del cliente como del proceso.

Así pues, los recursos se convierten en un elemento indispensable para la construcción de satisfacción del cliente. Al mismo tiempo la producción ayuda también a este establecer el ritmo acorde con sus capacidades, en un constante equilibrio. Siempre en busca de no afectar el entorno social ni la obtención de beneficios para las dos partes; podría describirse como un gana gana, que así debería ser percibido por las dos partes.

Según el autor, el justo a tiempo busca agrupar características homogéneas tanto de clientes como de productores y permitir el desarrollo de productos con características de éxito en mercados más extensos. También hace posible plantear varias características en la presentación, configuración, logística y hasta en la calidad, de acuerdo con las necesidades del cliente.

Pero no solo estos estudios buscan la optimización de los recursos para lograr la eficiencia organizacional, sino que otros plantean la optimización de los procesos, teniendo en cuenta que la logística de trasporte y optimizar la fabricación son elementos cruciales.

En la literatura, se encuentran estudios que abordan el problema sin considerar el almacenamiento temporal de las mercancías entrantes, su objetivo es minimizar la distancia recorrida por los medios de manipulación. Plantean, en su mayoría, el uso de una formulación tradicional para el problema y un algoritmo de rama. El modelo es un punto de referencia en la literatura que está presente en los estudios de autores como (Bermúdez y Cole, 2001; Zhu, Hahn, Liu y Guignard, 2009; Cohen 
y Keren, 2009; Guignard, Hahn, Pessoa y Da Silva, 2012).

Boysen, Fliedner y Scholl (2010) utilizan una metodología que plantea un enfoque dinámico y métodos heurísticos para reducir al mínimo el tiempo total empleado en la línea de camiones entrantes y salientes, proponiendo una optimización del proceso.

Chen y Lee (2009) desarrollaron un algoritmo de aproximación polinómica para minimizar la acumulación de producción a través de una línea de producción sin almacenamiento temporal. En particular, los autores proponen hacer una secuencia para la descarga y carga, así como implementar operaciones de agrupación y desagrupación para las mercancías entrantes y salientes, con el fin de minimizar la acumulación y retardos en el proceso.

Otros estudios, preocupados por los tiempos de desplazamiento en los entornos cada vez más caóticos, tratan de encontrar la secuencia de programación tanto para camiones entrantes como salientes, con el propósito de minimizar el total de tiempo de funcionamiento considerando almacenamiento para guardar objetos temporalmente cerca del cliente basados en los cálculos de consumo (Boloori, Fatemi y Zandieh, 2011).

La globalización tecnológica ha llevado a las empresas a maximizar los recursos y la tecnología, pero es demasiado cambiante y costosa. El uso de leasing, por ejemplo, y las empresas dedicadas y monopolizadas sobre la tecnología, han generado una mayor exigencia e inversión por parte de las empresas.

\section{Metodología}

El artículo hace una reflexión sobre la información más relevante de la metodología de logística justo a tiempo. Su objetivo es indagar sobre los elementos principales del tema, mediante una revisión exhaustiva de literatura. La revisión de artículos científicos busca profundizar en aspectos relevantes que podrían aportar a la comprensión del modelo o como mínimo a identificar los aspectos que no han sido examinados.

Se emplea una metodología cualitativa de revisión, donde por medio de una base de datos se indagan sobre construcciones de variables en la literatura. Por medio de esta, se plantea ubicar los términos más comunes que pueden tener alguna relevancia y merecer algún desarrollo para la comprensión a fondo del fenómeno observado.

Para este ejercicio, se revisaron 83 documentos obtenidos en bases de datos como Scopus, Redalyc y Scielo, se aplicaron algunos criterios de exclusión que permitieron a los investigadores ubicar una base general de 49 artículos para el desarrollo de este estudio. Lo anterior se constituye en un cuerpo de evidencia empírica considerable; para este fin, se plantea el análisis de fuentes primarias y secundarias.

\section{Sistemas de producción eficientes}

Para la literatura, un proceso de producción se podría definir como el conjunto de operaciones que transforma insumos de entrada en productos que le agrega valor en el proceso. Este valor se define como la transformación que le imprime una funcionalidad o utilidad para el usuario o cliente mediante su transformación. Con este propósito, se plantea una secuencia programada de actividades que son indispensables para la elaboración del producto final.

La optimización de los procesos, así como el eficiente uso de los recursos, se convierten en elemento clave para el éxito del producto. La eliminación de procesos e insumos innecesarios puede marcar la clave entre el éxito y el fracaso. Así es como "todo lo que no sea la cantidad 
mínima de equipos, materiales, piezas, espacio y tiempo del operario, que resulten absolutamente esenciales para añadir valor al producto" (Shingo, 1993, p. 54.) debe ser examinado en el proceso de producción ajustada donde se analizan los excesos de existencias, los plazos de producción, la inspección, el movimiento y el almacenaje de materiales, las transacciones o los rechazos. "La fabricación justo a tiempo puede dar buenos resultados en cualquier ambiente fabril, en cualquier industria. Más aún, ahora estamos viendo que también podemos aplicarlo a empresas no fabriles" (Hay, 2002, p. 12).

Algunos estudios, como el de Jamal y Sarker (1993), analizan el sistema justo a tiempo y plantean el uso eficiente de materias primas mediante un orden e introducen el concepto de tamaño del lote como un aspecto importante. Este trabajo, complementado al siguiente año por otro estudio, considera las bondades de una política de aprovisionamiento en cuanto a materiales mediante un proceso de producción en escalones centrado en un uso más racional de las existencias en bodega (Sarker y Parija, 1994).

Estos dos aportes revolucionan los inventarios de la producción consiguiendo excelentes resultados siendo artículos seminales en el área.

Aquí encontramos una variable muy importante por revisar: los costos fijos, que entran a desempeñar un importante papel en la cadena de suministros, dando paso a nuevos modelos, como el de programación lineal planteado por Chu (1995), donde ingresan variables como la de restricción de materiales y recursos que tienen en cuenta la demanda total, en busca de habilitar secuencias en la producción y optimizar agregada mente el sistema, en contraste con el modelo propuesto el año anterior. Esto generó, además, toda una filosofía empresarial de revisión del desempeño empresarial competitivo en la función productiva, así como el constante ajuste de procesos y el surgimiento de múltiples modelos de producción que se impregnaban de justo a tiempo y la planificación de requerimientos de manufactura.

Después de la revisión de literatura, se hace importante también incluir en la discusión la optimización de los tiempos y la maquinaria por usar, su eficiencia sistemática y los grados de oportunidad frente a los diversos mercados. Para poder analizar los procesos justo a tiempo, es necesario analizar los otros procesos planteados, con el fin de comprender sus diferencias y bondades. Comenzamos con el concepto de globalización que implica los adelantos en la comunicación y el transporte que plantean cambios durante los procesos productivos.

Golhar y Sarker (1992) exploraron con un control de inventarios donde las variables principales son la producción y el alistamiento en contravía con los intervalos de envíos y descubrieron que en determinadas condiciones era posible disminuir costos, así como el tamaño del envío.

Uno de los conceptos que se debe tener en cuenta es el planteado por los sistemas de planificación de requerimientos de materiales, que fue presentado inicialmente en un trabajo de Orlicky (1975) y posteriormente ratificado por Segerstedt (1996) quien por medio de fórmulas algebraicas planteó técnicas de optimización. Para el modelo justo a tiempo, es indispensable que, no solo la producción sea factible, sino que también se incluya una variable indispensable que asuma que debe tener un atractivo económico. Sobre este tema se encuentran trabajos como el de Chu (1995), Clark (2003) y Mula, Poler y Garcia (2006) quienes afirman que este tipo de optimización se puede utilizar por medio de una aproximación a la lógica difusa propuesta por Escudero y Kamesam (1993) que aplicaron la generación de escenarios para esto.

Se plantea la planificación de requerimientos de materiales como una herramienta dirigida a la producción que analiza las 
condiciones del entorno, para optimizar los procesos mediante modelos matemáticos que determinan los requerimientos de materiales, insumos y capacidad instalada para la eficiencia. Este modelo fue revisado por Yenisey (1998) donde propuso una revaluación de sus principios fundamentales. Posteriormente, fue examinado nuevamente por el mismo autor (Yenisey, 2006) y considera un modelo basado en la red de flujos. Aquí se tienen en cuenta los tiempos de suministros de materiales e insumos, se plantea una debilidad en el modelo original, pues existe una inflexibilidad en el tamaño de los lotes de producción, que siempre tienen un tamaño fijo, lo cual contrasta con el comportamiento del mercado que es variable.

Paralelamente, se desarrolló la teoría de restricciones desarrollada por el físico israelí Eliyahu Goldratt y Jeff Cox en 1993. Esta se centra en la correcta administración de los recursos más débiles de la organización, con el fin de convertirlos en una ventaja competitiva. Más adelante fue retomada por ( Drucker, 1998), quien agregó la importancia de enfocar las soluciones en los problemas críticos en un constante proceso de mejora continua.

Una restricción es considerada cualquier cosa que limita que la empresa logre su objetivo, es decir que impida que gane más dinero. Cuando se habla de mejoramiento continuo, se hace referencia al mejoramiento de los procesos, a la forma de solucionar los problemas, al análisis de los costos, al manejo del talento humano, a la programación efectiva de la producción, a la identificación correcta de cuál es la restricción de la empresa y cómo eso está afectando la satisfacción del cliente y el crecimiento económico de la empresa (González, Ortegón y Rivera, 2003, p. 125).

Por otra parte, Clark (2003) plantea las bondades de los modelos de red para la revisión de flujos, algo que es imposible de lograr con los modelos lineales.
La Teoría de las Restricciones invita a los administradores de empresas a concentrar sus esfuerzos en las actividades que tienen incidencia directa sobre la eficacia de la empresa como un todo, es decir, sobre los resultados globales. Para que el sistema empresarial funcione adecuadamente las operaciones deben ser estabilizadas, para ello es necesario identificar y alterar las políticas contraproducentes. Entonces, se hace conveniente crear un patrón o modelo que incluya no apenas conceptos, sino principios orientadores y prescripciones, con sus respectivas herramientas y aplicaciones. (Dettmer, 1997, p. 62)

En la teoría, se evidencia la presencia de varios tipos de restricciones:

- producción,

- mercados,

- insumos y materiales y

- logística políticas y legislativas.

Drucker (2002) plantea las cortas diferencias existentes entre las empresas y lo valioso de cualquier diferencial en los procesos de producción considerando la oportunidad y el valor agregado en el producto como una ventaja competitiva sin detrimento de la calidad percibida. Para esto, otros estudios (Yacobucci, 2017; Hernández, Roldán y Ruano, 2003) describen la logística como eje fundamental en la diferenciación de productos. Las dos visiones apuntan a añadir valor agregado al producto final.

Según Hay (2002), el justo a tiempo no debe ser visto como solo un programa para la producción eficiente, sino que, en general, ha de ser implementado para la mejora continua de procesos de producción. Si bien afecta los procesos de compras, se centra en la interacción con las diferentes dependencias que lo convierte en una filosofía que redunda en eficiencia. Este, finalmente, debe establecer prioridades en los procesos con una constante revisión. 
"A los clientes les interesa recibir su pedido completo y en el tiempo justo y a otros les interesa completo o que cumplan los requerimientos de la entrega" (Ballou, 2004, p. 8). Teniendo en cuenta que no todos los clientes son iguales, es importante revisar las expectativas de cada uno para poder prestar un servicio que sea percibido como un valor agregado.

Por su parte, Heskett (1978, p. 32) afirma que "la finalidad del método justo a tiempo es mejorar la capacidad de una empresa para responder adecuadamente a la notoria variación de la demanda y las necesidades específicas de los clientes" ${ }^{1}$. Para Kotler y Keller (2006, p. 2), "los clientes tienen unas expectativas en relación con el servicio y las empresas se centran en lograr la máxima satisfacción del cliente". Si bien el objetivo está en buscar impactar la percepción del consumidor obteniendo los mejores resultados, se debe tener en el panorama la maximización del negocio, el perfecto equilibrio entre lo que genera valor para el cliente y lo que produce valor para la empresa. Así pues, es indispensable tener en el radar variables de impacto como el tiempo de entrega, el cumplimiento, la calidad del producto, el lugar de entrega y el momento exacto de entrega, así como otros aspectos que generan valor en el momento de la entrega como la facturación y el servicio prestado.

Chase, Aquilano y Jacobs (2000) presentan evidencias de que todas estas variables están directamente relacionadas con la formulación estratégica y tienen incidencia directa en la competencia, teniendo en cuenta que el consumidor de hoy da por descartado el tema de calidad en los productos, pues esta ya se ha convertido en una obligación y no en un diferencial. Lo que también es ratificado por Skinner (1974) y posteriormente por Da Silveira (2005).

La filosofía justo a tiempo para Hay (2002) debe combinar las viejas formas de hacer las cosas con nuevas técnicas, con el propósito de renovar los procesos en las áreas que aún continúan haciendo las cosas de la manera tradicional. Para esto, teóricos como Chopra y Meindl (2007) consideran la existencia de tres macroprocesos que suministrarían la información necesaria a fin de aplicar correctamente un proceso de justo a tiempo. El primero sería la gestión de proveedores (suppliers relationship management), donde se controlan y gestionan todos los procesos con proveedores, en busca de oportunidades, siempre teniendo en cuenta las necesidades de nuestros clientes, lo cual permite mejorar nuestra negociación y hacerla más eficiente. El segundo es el proceso interno (internal supply chain management), que plantea la estrategia de integración entre las diferentes áreas de la producción, comercialización e investigación. En este proceso, la empresa debe desligarse de la tradición y encontrar las formas más eficientes. Aquí se incluyen las estrategias de outsourcing maquilas y producciones in house. Finalmente, gestión de relaciones con el cliente (customer relationship management) donde se buscan todos los puntos de contacto con el consumidor final; aquí se deben identificar las oportunidades de generación de valor para el producto y remitir la información a los otros dos procesos.

La revisión de literatura coincide en que a la luz de la teoría actual la definición se queda algo corta, puesto que se basa en una teoría antigua que debe ser aterrizada a nuestra época, a procesos de producción actuales, pero hace falta tener en cuenta un mayor número de variables que afectan el proceso. (Lefcovich, s. f.) plantea cinco variables que deberían ser revisadas: costo, calidad, servicio, flexibilidad e innovación.

La satisfacción del cliente se ha convertido en una meta principal de la actividad empresarial. El servicio al cliente debe ser definido, medido y gestionado (Hernández, Roldán y Ruano, 2003). Por su parte, Martínez y Lauzardo (2004, p. 8) señalan que "es indispensable analizar el comportamiento de los consumidores, teniendo en

\footnotetext{
${ }^{1}$ Las traducciones son nuestras.
} 
cuenta que en los últimos años los clientes han ido evolucionando y son ahora más demandantes, más conocedores, más sofisticados y deseosos de tomar sus propias decisiones". Los clientes desempeñan un papel importante y las compañías, independiente de su tamaño, deben trabajar constantemente para satisfacer sus necesidades e innovar de acuerdo con las exigencias del mercado.

Ahora los clientes toman decisiones más inteligentes en un medio donde la información está disponible más fácil. Las empresas deben trabajar constantemente en la identificación de sus necesidades con innovacion y eficiencia, pues ahora no es solo ofrecer un producto, sino que también se deben planear las mejores prácticas que generen valor agregado a la empresa y los consumidores

La logística como modelo de gestión en cualquier organización busca administrar estratégicamente la cadena de valor y los procesos de retorno asociados con esta. Lo anterior con el objetivo de alcanzar la eficiencia, la efectividad y la plena satisfacción de los grupos de interés (stakeholders). En este sentido, se asegura la perdurabilidad de la organización. (Trujillo y Guzmán, 2007, p. 97)

Así es como la mirada de estas variables y el desarrollo de estrategias busca desarrollar ventajas competitivas. Para Porter y Kramer (2002, p. 178), "las ventajas competitivas no se pueden entender o identificar considerando la compañía como un todo. Por lo tanto, las auténticas ventajas en costos y diferenciación se deben buscar en la cadena de actividades que una empresa realiza para poder otorgar valor a sus clientes". Según asegura (Boyett, 1999, p. 25), "las mejores ideas de los máximos pensadores de la administración, sugiere que los directivos han de reconducir el análisis estratégico detallado hacia la cadena de valor para después poder elegir la estrategia que más les convenga", lo cual no riñe con lo planteado por Porter y Kramer. El control se convierte en una propiedad

para establecer mecanismos a fin de identificar oportunidades. Se debe evaluar incluso hasta una reducción de eficiencia a corto plazo si esto llegara a implicar una ventaja mientras se ajustan los procesos.

\begin{abstract}
La logística se ocupa del proceso de planificación, operación y control del movimiento y almacenaje de mercancías, así como de los servicios informáticos asociados. En el mundo empresarial, la logística pretende manejar el flujo de productos desde el origen hasta la entrega al consumidor final. (Bastos, 2007, p. 59)
\end{abstract}

Según Hay (2002), el éxito en los procesos justo a tiempo está en la búsqueda de la simplicidad, de un correcto flujo de material que controle los procesos. La eficiencia en espacio, alquileres y optimización de áreas son aspectos importantes por tener en cuenta, que deben surgir del análisis de los diferentes procesos, así como propender a una correcta circulación de la información y constante búsqueda de la eficiencia y eficacia en los procesos y las posibilidades futuras del negocio.

\section{Discusión}

Es innegable que la logística integral debe ser atendida como un proceso holístico en los negocios. La competitividad depende de la capacidad de adaptación y comprensión de los entornos, así como de la identificación de oportunidades e innovación en los procesos.

La integración de los procesos logísticos en los objetivos estratégicos de la organización requiere una comunicación interfuncional entre las dependencias, lo cual no es posible sin un correcto posicionamiento de la función logística en la toma de decisiones. Este proceso debe contar con variables que impliquen la generación de valor a nuestros clientes como calidad, flexibilidad, confiabilidad, oportunidad, ubicación y precio correcto. 
La integración logística se hace necesaria en un ámbito geográfico extenso, para esto se plantea la necesidad de conformar redes sistémicas que se adapten a las oportunidades que ofrece esta nueva característica del entorno.

Un común denominador en la literatura consultada para este artículo se plantea la necesidad de involucrar técnicas complementarias al proceso de justo a tiempo; se hace énfasis en su enfoque holístico que implica múltiples dimensiones. Las futuras investigaciones se podrían plantear la necesidad de utilización de técnicas cualitativas y cuantitativas para la apropiación de las bondades de esta teoría a la luz de los cambios actuales en la demanda y los cambios de los consumidores inmersos en un mundo globalizado y con nuevos hábitos de consumo.

Las empresas deben estar en una constante vigilancia de buenas prácticas, preguntándose todo el tiempo por las mejores opciones. No obstante, las mejores prácticas tendrán fundamento en las necesidades de nuestros consumidores o potenciales clientes.

De esta revisión, podemos resaltar la falta de un conjunto exacto de indicadores que se han de tener en cuenta por parte de las empresas. Las limitaciones de costos, oportunidades, tiempo y disponibilidad hacen de cada empresa un contexto único que debe definir su rumbo por medio del análisis completo de su entorno. La elaboración de un modelo que permita la caracterización de una práctica logística integral como el justo a tiempo podría ser una oportunidad para desarrollar en el futuro.

Los textos coinciden en que el modelo justo a tiempo, que incluye una aceptación de la cadena de valor y la forma como se organiza la logística de los productos, el servicio al cliente, la logística inversa y la reducción de costos y la producción, implica recursos y una administración de negocios muy organizada.
Es evidente, de igual manera, la importancia para los autores de la eficiencia del tiempo, los recursos financieros y los trabajadores. Podríamos concluir que hacer más eficiente el proceso de producción y de calidad del producto es una preocupación más frecuente para los países más grandes, mientras que los países pequeños lo hacen más por la gestión de las materias primas, la globalización de mercados y la evolución tecnológica. Esto implica el cambio logístico, la búsqueda de oportunidades de negocio y de productos diferenciales.

La producción y los insumos, gracias a la globalización uno de los principales intereses de las empresas, actualmente es una de las pocas oportunidades para generar diferenciales. Las empresas deben optar por generar valor a sus productos, dando por descontada la calidad. Esto a la par de una dinámica estructural que derive en crecimiento y posicionamiento en el mercado mundial o local.

\section{Referencias}

Ballou, R. H. (2004). Logística: administración de la cadena de suministro (5. ${ }^{\mathrm{a}}$ ed.). México: Pearson Educación.

Bastos Boubeta, A. I. (2007). Distribución logística y comercial: la logística en la empresa. Madrid: Ideaspropias.

Bermúdez, R. y Cole, M. H. (2001). A genetic algorithm approach to door assignments in breakbulk terminals. Recuperado de http://citeseerx.ist.psu.edu/viewdoc/download?doi=10.1.1.595.5287\&rep=rep1\&type= pdf

Boloori Arabania, A. R., Fatemi Ghomia, S. M. T. y Zandiehb, M. (2011). Meta-heuristics implementation for scheduling of trucks in a crossdocking system with temporary storage. Expert Systems with Applications, 38(3), 1964-1979.

Boyett, J. (1999). Hablan los gurús: las mejores ideas de los máximos pensadores de la administración. Bogotá: Norma.

Boysen, N., Fliedner, M. y Scholl, A. (2010). Scheduling inbound and outbound trucks at cross docking terminals. OR Spectrum, 32(1), 135-161. 
Chase, R. B., Aquilano, N. y Jacobs, R (2000). Administración de producción y operaciones: manufactura y servicios. Bogotá: McGrawHill.

Chen, F. y Lee, C. Y. (2009). Minimizing the makespan in a two-machine cross-docking flow shop problem. European Journal of Operational Research, 193(1), 59-72.

Chopra, S. y Meindl, P. (2007). Management strategy (3. ${ }^{a}$ ed.). New Jersey: Prentice Hall.

Chu, S. C. (1995). A mathematical programming approach towards optimized master production scheduling. International Journal of Production Economics, 38(2-3), 269-279.

Clark, A. R. (2003). Optimization approximations for capacity constrained material requirements planning. International Journal of Production Economics, 84(2), 115-131.

Cohen, Y. y Keren, B. (2009). Trailer to door assignment in a synchronous cross-dock operation. International Journal of Logistics Systems and Management, 5(5), 574-590.

Dettmer, H. W. (1997). Goldratt's theory of constraints: A systems approach to continuous improvement. Milwaukee, Wisconsin: ASQ Quality Press.

Drucker, P. F. (1998). Management's new paradigms. Forbes Magazine, 10(2), 98-99.

Druker, P. F. (2002). Los desafíos de la gerencia para el siglo XXI. Bogotá: Norma.

Escudero, L. F. y Kamesam, P. V. (1993). planificación de requerimientos de materiales modelling via scenarios. En Optimisation in industry (pp. 101-111). Nueva York: John Wiley and Sons.

Goldratt, E. M. y Cox, J. (1993). La meta: un proceso de mejora continua. Monterrey: Castillo.

Golhar, D. Y. y Sarker, B. R. (1992). Economic manufacturing quantity in a just-in-time delivery system. Journal of Production Research, 30(5), 961-972.

González Gómez, J. A., Ortegón Mosquera, K. y Rivera Cadavid, L. (2003). Desarrollo de una metodología de implementación de los conceptos de teoría de restricciones (teoría de restricciones) para empresas colombianas. Estudios Gerenciales, 19(87), 27-49.

Guignard, M., Hahn, P. M., Pessoa A. A. y da Silva, D. C. (2012). Algorithms for the cross dock door assignment pro- blem. Recuperado de http://ai2-s2-pdfs.s3.amazonaws.com/be51/177a7e047d475b46e91e343006 b86f41d833.pdf

Magableh, G. M., Rossetti, M. D. y Mason, S. (2005). Modeling and analysis of a generic crossdocking facility. En Proceedings of the 37th conference on Winter simulation (pp. 1613-1620). Orlando, Florida: Winter Simulation Conference.

Hay, E, J. (2002). Justo a tiempo (just in time): la técnica japonesa que genera mayor ventaja competitiva (trad. M. Cárdenas). Bogotá: Norma.

Hernández Rodríguez, N. R., Roldán Ruenes, A. y Ruano Ortega, E. R. (2003). La logística y su papel en el desarrollo de las organizaciones. Santiago de Cuba: Universidad de Oriente.

Heskett, J. (1978). La logistique élément clé de la stratégie. Harvard-L'Expansion, 8, 53-65.

Jamal, A. M. M. y Sarker, B. R. (1993). An optimal batch size for a production system operating under a just-in-time delivery system. International Journal of Production Economics, 32(2), 255-260.

Kotler, P. y Keller, K. L. (2006). Dirección de marketing (12. ${ }^{a}$ ed.). México: Pearson Educación.

Lefcovich, M. (s. f.). Producción justo a tiempo para la competitividad total.Recuperado de http://www.monografias.com/trabajos17/competitividad-total/competitividad-total.shtml

Martínez Delgado, E. y Lauzardo Rico, J. (2004). El servicio al cliente: una necesidad imperante en la calidad de la industria. Cuba: Instituto Superior Politécnico José Antonio Echeverría.

Mula, J., Poler, R. y Garcia, J. P. (2006). planificación de requerimientos de materiales with flexible constraints: A fuzzy mathematical programming approach. Fuzzy Sets and Systems, 157(1), 74-97

Ohno, T. (1988). Toyota production system: Beyond large-scale production. Portland, Oregon: Productivity Press.

Orlicky, J. (1975). Material requirements planning. Londres: McGraw-Hill.

Porter, M. E. y Kramer, M. R. (2002). La ventaja competitiva de la filantropía corporativa. Harvard Business Review, 80(12), 49-62.

Sarker, B. y Parija, G. (1994). An optimal batch size for a production system operating under a fixed quantity, periodic delivery policy. Journal 
of the Operations Research Society, 45(8), 891-900.

Segerstedt, A. (1996). Formulas of planificación de requerimientos de materiales. International Journal of Production Economics, 46, 127136.

Shingo, S. (1993). El sistema de producción Toyota desde el punto de vista de la ingeniería. Madrid: Tecnologías de Gerencia y Producción.

Silveira, G. J. da (2005). Market priorities, manufacturing configuration, and business performance: an empirical analysis of the order-winners framework. Journal of Operations Management, 23(6), 662-675.

Skinner, W. (1974). The focused factory (vol. 52, pp. 113-121). Brighton, MA: Harvard Business Review.

Trujillo Dávila, M. A. y Guzmán Vásquez, A. (2007). La administración logística inmersa en las megatendencias empresariales. Universidad \& Empresa, 9(13), 91-112.

Yacobucci, G. (2017). Hacia una ética del desarrollo económico. Enfoques, 7(1), 27-33.

Yenisey, M. M. (1998). Flow-network approach to materials requirement planning. En Proceedings of the second International Symposium on Intelligent Manufacturing Systems (vol. 1, pp. 529-533). Sakarya, Turquía.

Yenisey, M. M. (2006). A flow-network approach for equilibrium of material requirements planning. International Journal of Production Economics, 102(2), 317-332.

Zhu, Y. R., Hahn, P. M., Liu, Y. y Guignard, M. (2009). New approach for the cross-dock door assignment problem. En Proceedings of the XLI Brazilian Symposium on Operations Research (pp. 45-50). Bahia, Brasil. 